\title{
Transient and permanent effects of suboptimal incubation temperatures on growth, metabolic rate, immune function and adrenocortical responses in zebra finches
}

\author{
Haruka Wada ${ }^{1, *, \ddagger}$, Buddhamas Kriengwatana ${ }^{1}$, Natalie Allen ${ }^{2}$, Kimberly L. Schmidt ${ }^{1}$, Kiran K. Soma ${ }^{3,4,5}$ and \\ Scott A. MacDougall-Shackleton ${ }^{1}$
}

\begin{abstract}
In birds, incubation temperature can vary by several degrees Celsius among nests of a given species. Parents may alter incubation temperature to cope with environmental conditions and/or to manipulate embryonic development, and such changes in incubation behavior could have long-lasting effects on offspring phenotype. To investigate short- and long-term effects of suboptimal incubation temperatures on survival and physiological functions in zebra finches, eggs were incubated at $36.2,37.4$ or $38.4^{\circ} \mathrm{C}$ for the entire incubation period. The post-hatch environment was identical among the treatment groups. We found that hatching success was lowest in the $38.4^{\circ} \mathrm{C}$ group, while post-hatch survival was lowest in the $36.2^{\circ} \mathrm{C}$ group. Incubation temperature had sex-specific effects on offspring phenotype: incubation temperatures affected body mass $\left(M_{\mathrm{b}}\right)$ but not physiological parameters of males and conversely, the physiological parameters but not $M_{\mathrm{b}}$ of females. Specifically, males from the $38.4^{\circ} \mathrm{C}$ group weighed significantly less than males from the $36.2^{\circ} \mathrm{C}$ group from the nestling period to adulthood, whereas females from different incubation temperature groups did not differ in $M_{\mathrm{b}}$. In contrast, females incubated at $36.2^{\circ} \mathrm{C}$ had transient but significantly elevated basal metabolic rate and adrenocortical responses during the nestling and fledgling periods, whereas no treatment effect was observed in males. Innate immunity was not affected by incubation temperature in either sex. These results suggest that a $1^{\circ} \mathrm{C}$ deviation from what is considered an optimal incubation temperature can lower offspring performance and offspring survival.
\end{abstract}

KEY WORDS: Development, Corticosterone, Pre-hatch, Stress, Altricial, Birds

\section{INTRODUCTION}

Environmental heterogeneity induces phenotypic variance among individuals as animals adjust their phenotype to match the environment. Environmental cues during development are particularly important because developing young typically display the greatest phenotypic plasticity. For oviparous species, incubation

\footnotetext{
${ }^{1}$ Advanced Facility for Avian Research, University of Western Ontario, London, Ontario, Canada N6G 1G9. ²Department of Psychology, University of Western Ontario, London, Ontario, Canada N6A 5C2. ${ }^{3}$ Department of Psychology, University of British Columbia, Vancouver, British Columbia, Canada V6T 1 Z4. ${ }^{4}$ Graduate Program in Neuroscience, University of British Columbia, Vancouver, British Columbia, Canada V6T 1Z4. ${ }^{5}$ Department of Zoology, University of British Columbia, Vancouver, British Columbia, Canada V6T 1 Z4.

*Present address: Department of Biological Sciences, 101 Rouse Life Science Building, Auburn University, AL 36849, USA.

${ }^{\ddagger}$ Author for correspondence (haruka@auburn.edu)
}

Received 15 September 2014; Accepted 7 July 2015 temperature greatly influences offspring phenotype (DuRant et al., 2013). In the few avian species with known incubation temperatures, the difference in incubation temperature between nests can be $3^{\circ} \mathrm{C}$ or more. For example, daytime incubation temperature of biparental zebra finches in the wild ranges from 34.9 to $38.5^{\circ} \mathrm{C}$ (Zann and Rossetto, 1991). An optimal incubation temperature is suggested to be around $37^{\circ} \mathrm{C}$ for zebra finches; it is the average incubation temperature in captive zebra finches (Vleck, 1981; Zann and Rossetto, 1991) with high embryo mass, residual yolk and growth efficiency (Olson et al., 2006). Thus, the observed variation indicates that not all parents incubate eggs at the optimal temperature for embryonic development. This is particularly true when environmental condition deteriorates; an artificial drop in the ambient temperature resulted in increased metabolic rate of the incubating zebra finches and a decline in incubation temperature (Nord et al., 2010). In such environmental conditions, parents face a trade-off between energetic needs of the parents and of the developing embryos, and can result in incubation temperatures that are not optimal for embryonic development.

Resulting changes in incubation temperature can have transient or long-term consequences on the offspring phenotype. Previous studies show that incubation temperature affects metabolic rates and the hypothalamic-pituitary-adrenal (HPA) axis function in birds for the first 2 weeks of life (DuRant et al., 2010; Nord and Nilsson, 2011): altricial blue tit (Cyanistes caeruleus) nestlings that hatched from eggs incubated at $35.0^{\circ} \mathrm{C}$ had significantly higher resting metabolic rates at $\sim 14$ days post-hatch (dph; hatch day is $0 \mathrm{dph}$ ) compared with nestlings hatched from eggs incubated at 36.5 or $38.0^{\circ} \mathrm{C}$ (Nord and Nilsson, 2011). Similarly, precocial wood duck (Aix sponsa) embryos that were incubated at $35.0^{\circ} \mathrm{C}$ expended significantly more energy during the hatching process compared with embryos incubated at 35.9 or $37.0^{\circ} \mathrm{C}$ (DuRant et al., 2011). The young hatched from eggs incubated at $35.0^{\circ} \mathrm{C}$ also had significantly higher baseline and stress-induced corticosterone levels compared with ducklings in the 35.9 or $37.0^{\circ} \mathrm{C}$ groups during the first 9 days of their lives (DuRant et al., 2010). These studies illustrate that incubation temperature has the potential to shape offspring phenotype in birds similar to maternal programming in mammals by licking and grooming behavior (Meaney, 2001) or through lactation (Fodor and Zelena, 2014).

One aspect of maternal programming in mammals is that changes to offspring physiology are often long-lasting or permanent (Fowden et al., 2006). If incubation temperature indeed shapes offspring phenotype in birds as maternal programming does in mammals, then impacts of incubation temperature should also endure. Surprisingly, this prediction has never been addressed. Therefore, we sought to shed light on this issue by empirically assessing the long-term consequences of variation in incubation temperature on offspring 


\section{List of symbols and abbreviations \\ ACTH adrenocorticotropic hormone \\ BMR basal metabolic rate \\ Dex dexamethasone \\ dph days post-hatch \\ HPA hypothalamic-pituitary-adrenal \\ $M_{\mathrm{b}} \quad$ body mass \\ PMR peak metabolic rate}

phenotype. Towards this end, we artificially incubated zebra finch eggs at low $\left(36.2^{\circ} \mathrm{C}\right)$, control $\left(37.4^{\circ} \mathrm{C}\right)$ and high $\left(38.4^{\circ} \mathrm{C}\right)$ temperatures. We then measured pre- and post-hatch survival, growth and vital physiological functions, including metabolic rates, immune and endocrine function. We hypothesized that when incubation temperature deviates from optimal, as occurs in cases of egg neglect or inefficient incubation, it would lower offspring performance and survival, leading to reduced parental fitness.

\section{RESULTS}

\section{Loss of egg mass}

During the first 12 days of incubation, eggs incubated at 36.2, 37.4, and $38.4^{\circ} \mathrm{C}$ lost $8.20 \%, 9.62 \%, 11.86 \%$ of mass, respectively. Both incubation temperature $\left(F_{2,144}=3.46 ; P=0.034\right)$ and incubation duration (covariate; $P<0.001$ ) had a significant effect on egg mass loss (Table 1). The post hoc analysis showed that eggs incubated at $38.4^{\circ} \mathrm{C}$ lost significantly more mass compared with controls at $37.4^{\circ} \mathrm{C}$. On average, eggs incubated at $37.4^{\circ} \mathrm{C}$ lost $9.1 \pm 0.41 \mathrm{mg}$ of their mass per day during the first 12 days of incubation compared with $7.8 \pm 0.24 \mathrm{mg}$ in the $36.2^{\circ} \mathrm{C}$ group and $11.3 \pm 0.41 \mathrm{mg}$ in the $38.4^{\circ} \mathrm{C}$ group

\section{Incubation duration and hatchling mass}

Incubation duration increased at lower temperatures $\left(F_{2,148}=239.5\right.$; $P<0.001$; Table 1). Specifically, eggs incubated at $36.2^{\circ} \mathrm{C}$ took 14.9 days to hatch, which was significantly longer than eggs incubated at $37.4^{\circ} \mathrm{C}$ with an average of 13.6 days, which was, in turn, significantly longer than eggs incubated at $38.4^{\circ} \mathrm{C}$ with an average of 12.8 days. Since embryos are ectotherms, we calculated degree-days by multiplying incubation duration by the incubation temperature to more appropriately describe the timing of hatching. If the difference in hatching duration was solely due to incubation temperature, degree-days among the treatment groups would be similar. However, they were not: time to hatching still increased with decreasing incubation temperature, with means of $537.9 \pm 1.9$, $507.6 \pm 2.87,491.3 \pm 2.52$ degree-days for the $36.2,37.4$ and $38.4^{\circ} \mathrm{C}$ groups, respectively. This difference in degree-days among the three incubation temperatures suggests that additional factors, such as embryonic metabolic rates, differ among the treatment groups.

Hatchlings weighed an average of $0.823,0.816$ and $0.824 \mathrm{~g}$ in the $36.2,37.4$ and $38.4^{\circ} \mathrm{C}$ groups, respectively. When initial egg mass was accounted for (covariate; $P<0.001)$, hatchling body mass $\left(M_{\mathrm{b}}\right)$ did not differ among the treatment groups $\left(F_{2,147}=0.03 ; P=0.97\right.$; Table 1). Thus, incubation temperature did not affect $M_{\mathrm{b}}$.

\section{Survival}

Eggs incubated at the control temperature of $37.4^{\circ} \mathrm{C}$ had $84.6 \%$ hatching success (determined as percentage of fertilized eggs that hatched in each treatment), where $6.2 \%$ died during embryonic development and 9.2\% died during the process of hatching (Fig. 1). Similar hatching success of $80.3 \%(8.5 \%$ and $11.3 \%$ died during embryonic development and during the process of hatching, respectively) was observed in the $36.2^{\circ} \mathrm{C}$ group $\left(\chi_{(1)}^{2}=0.443\right.$, $P=0.506)$. In contrast, the $38.4^{\circ} \mathrm{C}$ group only had $59.1 \%$ hatching success $\left(\chi_{(1)}^{2}=12.04, P=0.001\right.$ compared with controls). Most of the mortality occurred during embryonic development $39.4 \%$ died during embryonic development versus $1.5 \%$ during the process of hatching).

Unlike hatching success, the $36.2^{\circ} \mathrm{C}$ temperature group had the lowest post-hatch survival compared with both $37.4^{\circ} \mathrm{C}$ and $38.4^{\circ} \mathrm{C}$ temperature groups $\left(\chi_{(1)}^{2}=5.22, P=0.022\right.$ in comparison with $37.4^{\circ} \mathrm{C}$; $\chi_{(1)}^{2}=7.33, P=0.007$ in comparison with $38.4^{\circ} \mathrm{C}$; Fig. 2 ). In the $36.2^{\circ} \mathrm{C}$ temperature group, $17.5 \%$ died during the nestling period $(21 \mathrm{dph}$ or younger) compared with $5.2 \%$ and $0 \%$, respectively, in the 37.4 and $38.4^{\circ} \mathrm{C}$ temperature groups. Most of these deaths occurred before nestlings were 3 days old. Another peak occurred between 32 and $38 \mathrm{dph}$ (around the time of nutritional independence), when $7.5 \%$ of the $36.2^{\circ} \mathrm{C}$ temperature group died whereas none of the 37.4 or $38.4^{\circ} \mathrm{C}$ groups died. In total, 11 birds died from the $36.2^{\circ} \mathrm{C}$ group after hatching whereas 3 and 1 bird died from the 37.4 or $38.4^{\circ} \mathrm{C}$ groups, respectively. When averaged across pre- and post-hatch period, overall survival for $36.2,37.4$ and $38.4^{\circ} \mathrm{C}$ groups was $76.4,88.4$, and $77.9 \%$, respectively.

\section{Nestling growth}

For both males and females, $M_{\mathrm{b}}$ of the genetic parents, measured just prior to breeding, had a significant effect on the offspring $M_{\mathrm{b}}$ (covariate; females: $P<0.001$; males: $P=0.002$ ). Incubation temperature alone affected $M_{\mathrm{b}}$ in males $\left(F_{2,50}=4.30 ; P=0.019\right)$ but not in females $\left(F_{2,36}=0.14 ; P=0.87\right.$; Fig. 3A,C). Post hoc analysis showed that male nestlings from the $38.4^{\circ} \mathrm{C}$ temperature weighed less than males from the $36.2^{\circ} \mathrm{C}$ temperature group (Fig. 3C,D). There was no interaction between age and incubation temperature treatment for either sex (age, females: $F_{8,288}=0.77 ; P=0.63$; males: $F_{8,400}=4.53 ; \quad P<0.001$; age $\times$ treatment, females: $F_{16,288}=0.59$; $P=0.89$; males: $F_{16,400}=0.89 ; P=0.59$ ). Interestingly, nestlings from both $36.2^{\circ} \mathrm{C}$ and $38.4^{\circ} \mathrm{C}$ weighed less than controls at 2 and $5 \mathrm{dph}$ for both sexes (Fig. 3B,D). Female nestlings from $36.2^{\circ} \mathrm{C}$ dropped to $86 \%$ of mean control mass at $2 \mathrm{dph}$ whereas female nestlings from $38.4^{\circ} \mathrm{C}$ dropped to $89 \%$ of mean control mass at $5 \mathrm{dph}$. Females from both temperature groups recovered to nearly $100 \%$ of mean control mass by $10 \mathrm{dph}$. Similarly, male nestlings from $36.2^{\circ} \mathrm{C}$ dropped to

Table 1. Egg measures and survival in offspring incubated at $36.2^{\circ} \mathrm{C}, 37.4^{\circ} \mathrm{C}$ or $38.4^{\circ} \mathrm{C}$

\begin{tabular}{|c|c|c|c|c|}
\hline \multirow[b]{2}{*}{ Variable } & \multicolumn{3}{|c|}{ Incubation temperature } & \multirow[b]{2}{*}{$P$ value } \\
\hline & $36.2^{\circ} \mathrm{C}$ & $37.4^{\circ} \mathrm{C}$ & $38.4^{\circ} \mathrm{C}$ & \\
\hline Egg mass loss (\%) & $8.20 \pm 0.22(56)$ & $9.62 \pm 0.43(54)$ & $11.86 \pm 0.42(36)$ & 0.034 \\
\hline Incubation duration (days) & $14.86 \pm 0.05(57)$ & $13.56 \pm 0.08(55)$ & $12.79 \pm 0.07(39)$ & $<0.001$ \\
\hline Hatch mass $(\mathrm{g})$ & $0.823 \pm 0.013(57)$ & $0.816 \pm 0.012(55)$ & $0.824 \pm 0.011(39)$ & 0.968 \\
\hline Hatchling success (\%) & $80.28(71)$ & $84.62(65)$ & $59.09(66)$ & see text \\
\hline Post-hatch survival (\%) & $72.50(40)$ & $92.11(38)$ & $96.77(31)$ & see text \\
\hline
\end{tabular}

Values are mean \pm s.e.m. $(N)$. 


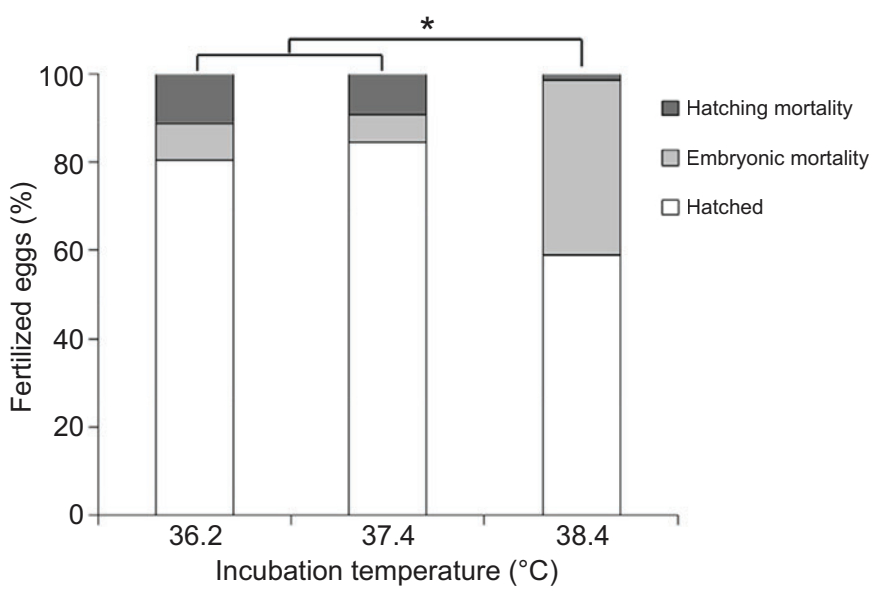

Fig. 1. Proportion of fertilized eggs that successfully hatched in zebra finch eggs incubated at $36.2,37.4$ or $38.4^{\circ} \mathrm{C}$. The asterisk indicates a statistical significance $(P<0.05)$.

$91 \%$ while male nestlings from $38.4^{\circ} \mathrm{C}$ dropped to $83 \%$ of mean control mass at $2 \mathrm{dph}$. Although $M_{\mathrm{b}}$ of male nestlings from $36.2^{\circ} \mathrm{C}$ recovered by $5 \mathrm{dph}$, male nestlings from $38.4^{\circ} \mathrm{C}$ recovered slowly to only $90 \%$ of mean control mass at $10 \mathrm{dph}$ and continued to be $\sim 96 \%$ of mean control mass throughout adulthood.

\section{Reactivity of the HPA axis}

At $16 \mathrm{dph}, M_{\mathrm{b}}$ of the individual did not affect baseline corticosterone or integrated adrenocortical response in either sex (females, baseline: $F=0.56, P=0.46$, integrated response: $F=1.00$, $P=0.32$; males, baseline: $F=0.28, P=0.60$, integrated response: $F=0.005, P=0.95)$. In males, incubation temperature did not affect nestling baseline $\left(F_{2,51}=0.28 ; P=0.76\right)$ or integrated adrenocortical response $\left(F_{2,51}=0.06 ; P=0.94\right.$; Table 2 , Fig. 4$)$. In contrast, females from the $36.2^{\circ} \mathrm{C}$ group had significantly higher adrenocortical responses compared with females from the $37.4^{\circ} \mathrm{C}$ or $38.4^{\circ} \mathrm{C}$ groups (baseline, $F_{2,36}=0.97 ; P=0.39$; integrated adrenocortical responses, $F_{2,36}=3.67 ; P=0.036$ ).

When birds reached adulthood, the treatment effect on the adrenocortical response disappeared. In both sexes, there was no effect of incubation temperature on baseline or integrated adrenocortical response (Table 2). Furthermore, incubation temperature did not have a significant effect on the integrated

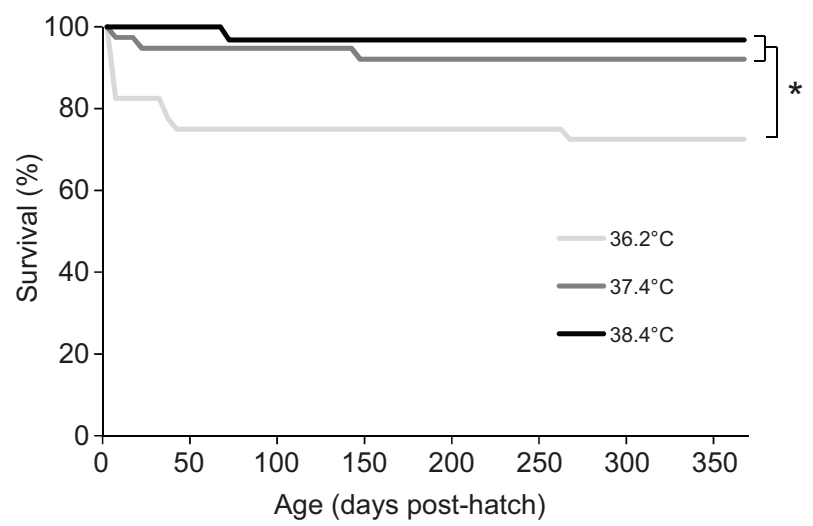

Fig. 2. Post-hatch survival of zebra finches hatched from eggs incubated at $36.2,37.4$ or $38.4^{\circ} \mathrm{C}$. Zebra finch nestlings fledge and reach nutritional independence around post-hatch day 21 and 35, respectively, and reach sexual maturity around 90 days after hatching. ${ }^{*} P<0.05$. response against administration of adrenocorticotropic hormone (ACTH) or dexamethasone (Dex). $M_{\mathrm{b}}$ at $266 \mathrm{dph}$ was marginally correlated with integrated Dex response in males $(F=3.87$, $P=0.055)$.

\section{Immune function}

Incubation temperature did not affect microbicidal capacity against Candida albicans or Escherichia coli in either sex (Table 2). No effect of incubation temperature was observed either during nestling period or in adulthood.

\section{Metabolic rates}

In females, incubation temperature significantly affected the basal metabolic rate (BMR) at $25 \mathrm{dph}$ where the $36.2^{\circ} \mathrm{C}$ females had a significantly higher BMR compared with $37.4^{\circ} \mathrm{C}$ females (Fig. 5, Table 2). The effect of incubation temperature on BMR was not observed at $177 \mathrm{dph}$, suggesting that the effect on BMR was shortlived. In males, there was no significant effect of the incubation temperatures on the BMR on either age. Similarly, incubation temperature did not affect peak metabolic rates (PMRs) in either sex.

\section{DISCUSSION}

The present study aimed to determine whether avian incubation temperature programs offspring vital physiological functions and influences offspring survival. In summary, we found that hatching success was lowest in the $38.4^{\circ} \mathrm{C}$ group while post-hatch survival was lowest in the $36.2^{\circ} \mathrm{C}$ group, ultimately leading to comparable survival rates in the low- and high-temperature groups by the end of the experiment (which were both lower than that of the control group). We also found that suboptimal incubation temperature affected males and females differently. Males from the $38.4^{\circ} \mathrm{C}$ group weighed less compared with males from the $36.2^{\circ} \mathrm{C}$ group throughout development and as adults, but no treatment effect was observed in regards to functioning of the HPA axis, innate immunity or metabolic rate. In contrast, females had similar $M_{\mathrm{b}}$ regardless of the temperature treatment but females from the $36.2^{\circ} \mathrm{C}$ group had a significantly higher adrenocortical response and BMR compared with females at 37.4 and $38.4^{\circ} \mathrm{C}$ during nestling and fledgling stages, respectively. However, these treatment effects disappeared in adulthood. Immune function in females was not affected by incubation temperature.

\section{High incubation temperature reduces hatching success whereas low incubation temperature reduces post-hatch survival}

It is thought that a shorter developmental period is favored when predation pressure is high (Case, 1978; Bosque and Bosque, 1995; Remeš and Martin, 2002; Martin and Briskie, 2009). Thus, parents may increase incubation temperature in an attempt to accelerate embryonic development. At the same time, markedly high incubation temperatures, typically above $40^{\circ} \mathrm{C}$, can cause malformations in the central nervous system (Peterka et al., 1996; Krausova and Peterka, 2007), increase production of reactive oxygen species (ROS) (Sakatani et al., 2004) and are considered lethal (Lundy, 1969; Conway and Martin, 2000). Even though $38.4^{\circ} \mathrm{C}$ is within the range of incubation temperature found in captive and free-living zebra finches (Zann and Rossetto, 1991), it is possible that this temperature increases abnormal mitotic activity (Sulik et al., 1988), production of ROS or the risk for malformation during embryogenesis, leading to the higher embryonic mortality seen in this study.

In contrast to the effects of high incubation temperature, reduced post-hatch survival in the low-temperature group may be due to low 

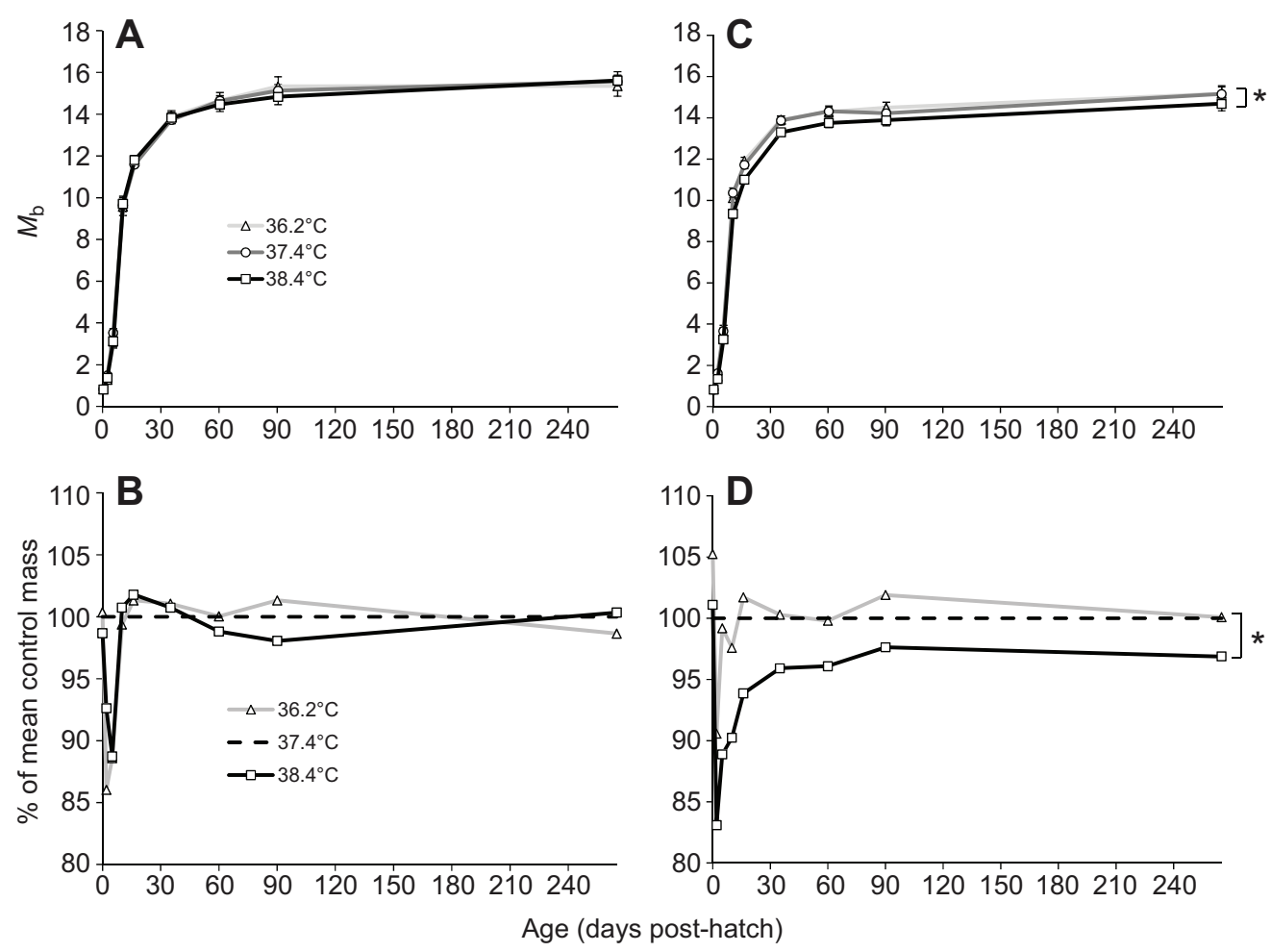

Fig. 3. Changes in body mass of zebra finches hatched from eggs incubated at $36.2,37.4$ or $38.4^{\circ} \mathrm{C}$ over the course of their lifetime.

$(A, B)$ Female birds. $(C, D)$ Male birds. $\mathrm{B}$ and $\mathrm{D}$ show percentage $M_{\mathrm{b}}$ in the 36.2 and $38.4^{\circ} \mathrm{C}$ groups compared with the mean $M_{\mathrm{b}}$ in the $37.4^{\circ} \mathrm{C}$ group. Error bars indicate \pm s.e.m. around the mean. Sample size at the end of the experiment ( $265 \mathrm{dph})$ was $21,14,19$ for males and $8,21,11$ for females incubated at $36.2,37.4$ and $38.4^{\circ} \mathrm{C}$, respectively. ${ }^{*} P<0.05$. residual energy at hatching. Higher temperature and shorter incubation period are linked to reduced yolk utilization in pine snakes (Burger et al., 1987), Australian brush turkeys (Eiby and Booth, 2009) and zebra finches (Olson et al., 2006). Conversely, low incubation temperature raises energy expenditure during development. In wood ducks for instance, total energy expenditure was similar among low, medium and high incubation temperature groups before the start of pipping; however, between pipping and hatching, embryos from the low-temperature group consumed more energy than those from the high and medium groups (DuRant et al., 2011). Olson et al. (2006) found that periodic cooling during the incubation period resulted in lighter embryos and less yolk reserve on embryonic day 12. Moreover, the mass-specific metabolic rates at embryonic day 12 were higher in the embryos that experienced periodic cooling compared with controls that were incubated at a constant temperature of $37.5^{\circ} \mathrm{C}$. These results suggest that a cooler temperature increases the energetic demand of embryos during development and requires yolk consumption beyond that of optimal incubation temperature (Olson et al., 2006). Since we measured percentage loss of egg mass at embryonic day 12 for all the temperature groups but did not measure residual yolk mass at hatching, it is difficult to compare the residual energy available at hatching. However, based on the data in reptiles and birds mentioned above, it is likely that embryos and hatchlings from the $36.2^{\circ} \mathrm{C}$ group used more yolk and hatched with lower residual yolk reserve compared with 37.4 or $38.4^{\circ} \mathrm{C}$ groups. In fact, young from the $36.2^{\circ} \mathrm{C}$ group suffered the highest mortality, particularly when the nestlings were between 1 and 3 days old. As all foster nests contained nestlings from all three treatment groups, the observed mortality in the early nestling stage may be due to a disadvantage in competing for food caused by low energy stores in the first days of life after hatching.

\section{High-temperature males weigh less throughout their lives}

Previous studies showed that hatchling $M_{\mathrm{b}}$ is positively correlated with incubation temperature. In precocial wood ducks, hatchling
$M_{\mathrm{b}}$ decreased with decreasing incubation temperature (Hepp et al., 2006, but also see DuRant et al., 2010). This is due to the increased energy expenditure during embryonic development at low incubation temperature (DuRant et al., 2011). In altricial zebra finches, periodic cooling to $20^{\circ} \mathrm{C}$ as opposed to constant incubation temperature of $37.5^{\circ} \mathrm{C}$ reduced $M_{\mathrm{b}}$ on embryonic day 12 without an impact on body size (Olson et al., 2006, 2008). In contrast to the studies above, we did not observe any effect of incubation temperature on hatchling $M_{\mathrm{b}}$. However, $M_{\mathrm{b}}$ in males from the $38.4^{\circ} \mathrm{C}$ group was lower after hatching compared with males from the $36.2^{\circ} \mathrm{C}$ and the effect persisted into their adulthood. This difference in $M_{\mathrm{b}}$ among treatment groups was largely due to the treatment difference in lean mass rather than lean and fat mass combined (H.W., B.K. and S.A.M.-S., unpublished data). As the quantitative magnetic resonance instrument we used to measure lean and fat mass does not detect skeletal or keratin-based structures (Guglielmo et al., 2011), the higher than optimal incubation temperature probably reduced muscle and organ mass in male offspring. Since BMR did not differ in males during fledgling or adult stages, metabolic rates do not contribute to the reduced muscle and/or organ mass. It is possible that males hatched from $38.4^{\circ} \mathrm{C}$ had suppressed begging, appetite or competitive ability, which reduced food intake. Whether the reduced $M_{\mathrm{b}}$ is an adaptation to the suboptimal temperature is not known. Further study is needed to test whether the low $M_{\mathrm{b}}$ in the high-temperature group enhances survival, reproductive performance and competitive ability relative to individuals with high $M_{\mathrm{b}}$ in a warm environment. It is worth noting that nestlings from both 36.2 and $38.4^{\circ} \mathrm{C}$ weighed less in the early nestling period compared with $37.4^{\circ} \mathrm{C}$ nestlings, regardless of sex (Fig. 3 ). In the statistical analysis, all birds that died before the juvenile period were excluded. Thus, this reduction in $M_{\mathrm{b}}$ at an early nestling period reflects a tangible and common difference in $M_{\mathrm{b}}$ between the optimal and suboptimal incubation temperature. 


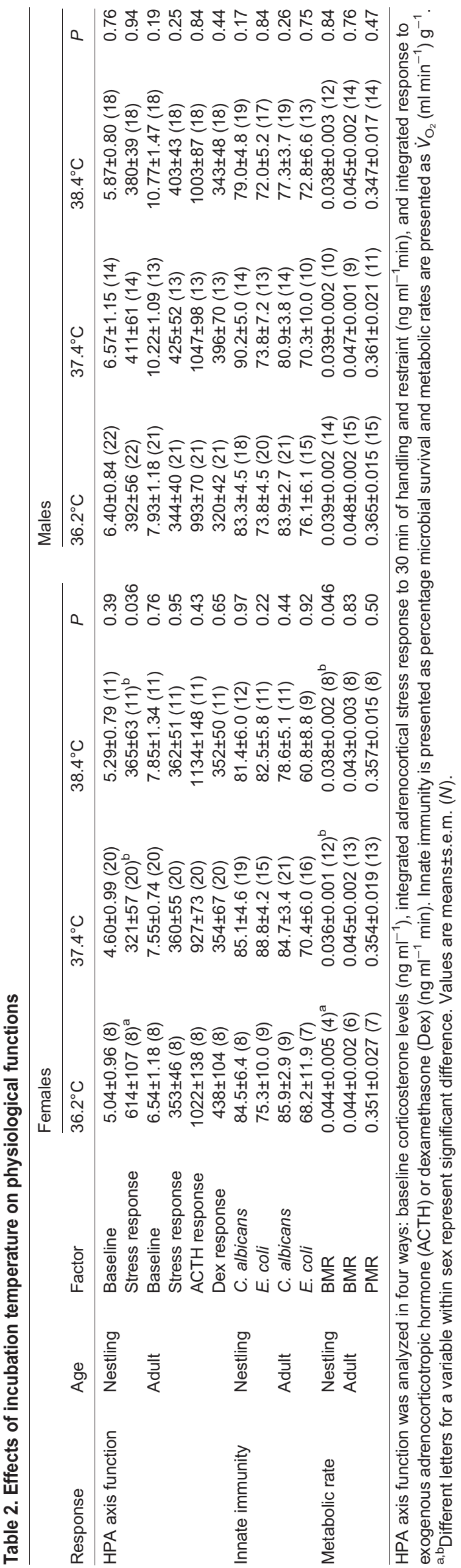

\section{Low-temperature females show a transient increase in adrenocortical response and BMR}

In altricial birds, a lower than optimal incubation temperature increases metabolic rates during embryonic development as well as in the nestling period (Olson et al., 2006; Nord and Nilsson, 2011). We observed that female fledglings from the $36.2^{\circ} \mathrm{C}$ group had a relatively high BMR compared with fledglings hatched from $37.4^{\circ} \mathrm{C}$. Our results indicate that the incubation temperature can affect BMR beyond the nestling stage. Lower than optimal incubation temperature also elevated adrenocortical responses in female nestlings. Similar patterns in the adrenocortical responses are reported in wood ducks, where ducklings from low incubation temperature exhibited elevated baseline and adrenocortical response compared with intermediate (with highest hatching success and post-hatch survival) and high incubation temperature at 2 and $9 \mathrm{dph}$ (DuRant et al., 2010). In our study, these effects on the HPA axis function and metabolism were observed for 16 and $25 \mathrm{dph}$, respectively. Offspring from the $36.2^{\circ} \mathrm{C}$ group suffered mortality at nutritional independence, approximately around $35 \mathrm{dph}$. Although we did not have a large enough sample size to test the relationship between treatment-related mortality and BMR or HPA axis function, further investigation into this relationship is warranted.

\section{Conclusion}

In summary, a $1{ }^{\circ} \mathrm{C}$ increase in incubation temperature reduced hatching success, whereas a $1.2^{\circ} \mathrm{C}$ decrease in incubation temperature reduced post-hatch survival. Suboptimal incubation temperatures also transiently, but significantly, elevated metabolic rate and adrenocortical responses and permanently reduced $M_{\mathrm{b}}$ in a sex-specific manner. These results therefore demonstrate that deviations as small as $1^{\circ} \mathrm{C}$ from optimal incubation temperature can have both short-term and permanent effects on offspring phenotypes in zebra finches. Since incubation temperature serves as an important source of phenotypic variability of offspring, it is imperative to assess the effects of environment, e.g. climate change, on incubation temperature and offspring phenotype in oviparous species. Whether or not the resulting phenotype is an adaptation to the forecasted environment or developmental constraint merits further investigation.

\section{MATERIALS AND METHODS Animal husbandry}

Zebra finches used in this experiment were obtained from our breeding colony at the Advanced Facility for Avian Research, University of Western Ontario, London, Canada. Animal husbandry and experimental protocols were approved by the Animal Use Subcommittee and followed the guidelines of the Canadian Council on Animal Care. From October 2010 to July 2011 , animals were kept in $36 \times 43 \times 42 \mathrm{~cm}$ cages with an external nest box $(20 \times 13.5 \times 13.5 \mathrm{~cm})$ attached to each cage. The room was kept at $22^{\circ} \mathrm{C}$ on $14 \mathrm{~h}: 10 \mathrm{~h}$ light:dark cycle. All pairs had access to seed (Living World premium finch seed, Mansfield, MA; $11.0 \%$ protein, $5.9 \%$ lipid), water, grit and cuttlebone ad libitum from pairing to rearing. In addition, protein-rich egg mixture (hardboiled chicken eggs, cornmeal and white bread) was provided daily to the pair from pairing to when the oldest nestling reached 35 dph.

\section{Manipulation of incubation temperature}

Based on variation observed in captive zebra finches, we chose three incubation temperatures: high, control and low. To manipulate incubation temperature, we incubated zebra finch eggs in three Brinsea Octagon 20 Advance EX incubators (Brinsea Products Inc. Titusville, FL, USA). Prior to the start of the experiment, all incubators were calibrated to a single reference thermometer (VWR ASTM thermometer, $34 / 42^{\circ} \mathrm{C}, \pm 0.1{ }^{\circ} \mathrm{C}$ accuracy; cat. no. 61126-943). Throughout the 


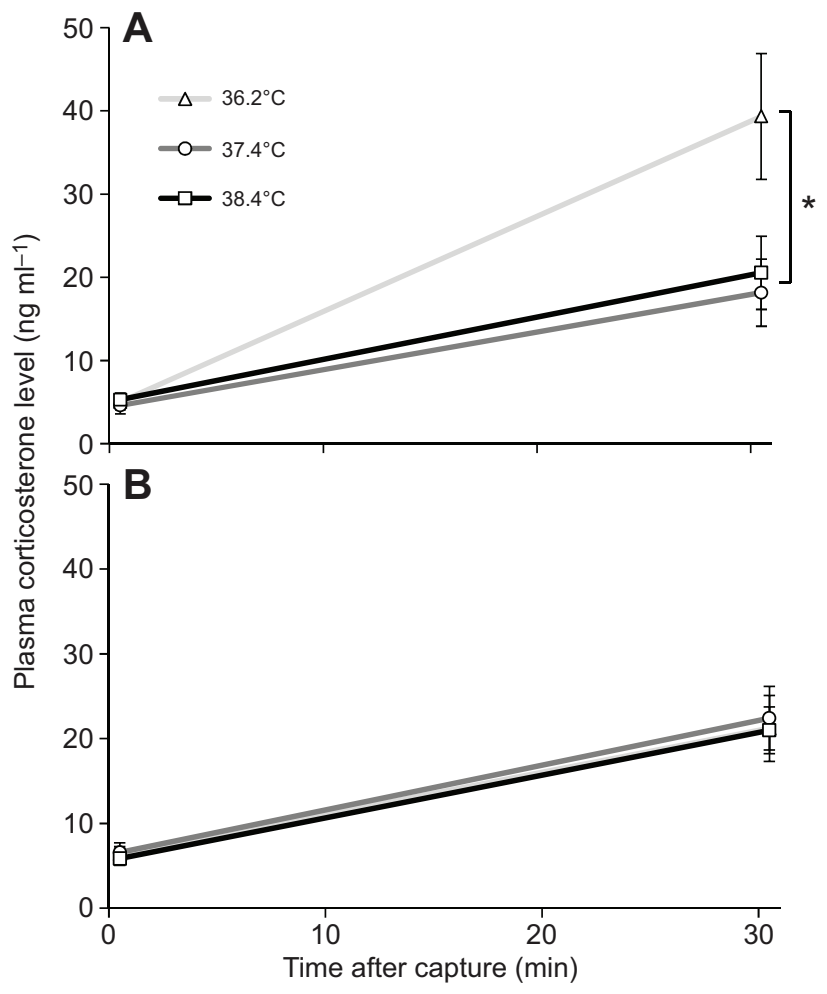

Fig. 4. Adrenocortical responses of zebra finches at 16 days post-hatch. (A) Female birds. (B) Male birds. Error bars indicate \pm s.e.m. around the mean. ${ }^{*} P<0.05$.

experiment, the temperature of each incubator at two positions within the row containing eggs was measured for 90 min weekly using the reference thermometer. Mean $( \pm$ s.e.m.) temperatures in low, control, and high incubators were $36.20 \pm 0.07(N=7), 37.42 \pm 0.08(N=7)$ and $38.40 \pm 0.07^{\circ} \mathrm{C} \quad(N=7)$, respectively, with $0.48 \%$ mean coefficient variation between the two positions. Relative humidity was set to $55 \%$ in all three incubators.

A total of 202 fertilized eggs from 26 breeding pairs was used in this experiment. Each nest box was checked daily and newly laid eggs were distributed to one of the three incubation temperatures. The first laid eggs were randomized and consecutive eggs were systematically allocated among the incubators so that an equal number of eggs from each nest were distributed among the three treatments and laying order was balanced among the treatments. Incubators were checked multiple times a day for new hatchlings. Hatchlings were placed into a nest with foster parents until each nest contained 5 or 6 nestlings comprising nestlings from all three treatment groups. To minimize mortality unrelated to the

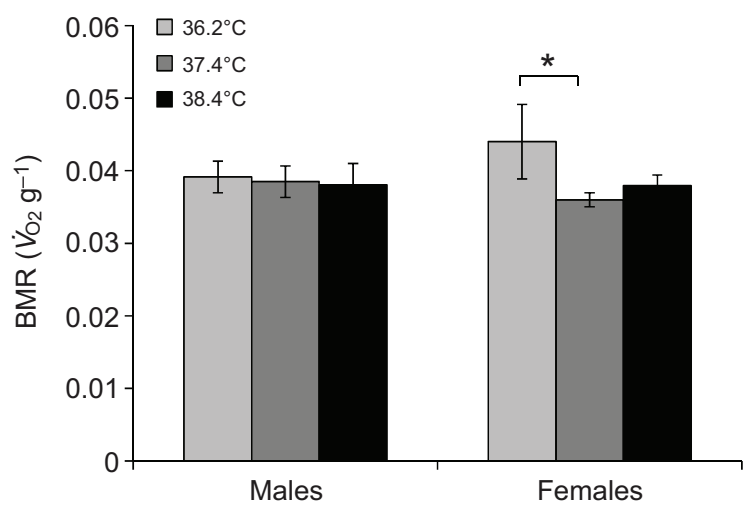

Fig. 5. Basal metabolic rates of zebra finches at 25 days post-hatch. Error bars indicate \pm s.e.m. around the mean. ${ }^{*} P<0.05$. temperature treatment, we minimized the age difference among foster siblings; all nests had nestlings that were no more than 1 day apart in hatch day except for 2 out of 22 nests that had a 2 day age difference. Any nests that had fewer than 5 nestlings in a nest at the beginning of the nestling period or did not have nestlings from all three treatment groups were excluded from further analysis.

\section{Pre-hatch measures on eggs}

Eggs were weighed to the nearest mg on the day they were laid and on the 12 th day of incubation to determine egg mass loss during incubation. All eggs were candled to assess whether the egg was fertilized. Only fertilized eggs were used in this study. For those fertilized eggs that failed to hatch, eggs were candled again to determine whether mortality occurred during embryonic development or during hatching process. The number of days for eggs to hatch was recorded as incubation duration.

\section{Nestling growth}

On the day of hatch $(0 \mathrm{dph})$, hatchlings were weighed to the nearest $\mathrm{mg}$. Nestlings were weighed to the nearest $0.1 \mathrm{~g}$ on $2,5,10,16,35,60$ and $90 \mathrm{dph}$ between $09: 30 \mathrm{~h}$ and 12:30 $\mathrm{h}$ before egg food was provided (see Fig. 6 for the timeline). The final $M_{\mathrm{b}}$ was measured on one day between 09:15 $\mathrm{h}$ and 14:15 $\mathrm{h}$ when birds were, on average, 266 $\pm 6.7 \mathrm{dph}$ $( \pm$ s.d.).

\section{Functioning of the hypothalamic-pituitary-adrenal axis}

We measured the functioning of the HPA axis in 4 ways: baseline corticosterone levels $\left(\mathrm{ng} \mathrm{ml}^{-1}\right)$ as well as adrenocortical response to standardized capture and handling stress, ACTH challenge and Dex suppression test (integrated response, $\mathrm{ng} \mathrm{m}^{-1} \mathrm{~min}$, see the statistical analysis section for details). We collected blood samples from individual birds by puncturing the brachial vein with a 26-gauge needle and collecting blood into heparinized microhematocrit tubes. All blood samples were immediately refrigerated. After centrifugation, plasma was extracted and frozen within $6 \mathrm{~h}$ of sample collection. Baseline blood

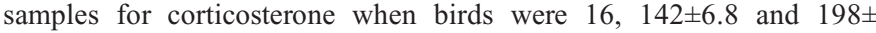
$5.9 \mathrm{dph}$ were obtained between 09:00 h and 11:05 h, within $3 \mathrm{~min}$ of entering the animal room. We measured adrenocortical responses to a stress using a standardized capture and handling protocol (Wingfield, 1994). After the baseline sample was collected, birds were restrained in an opaque bag, and a second blood sample was collected $30 \mathrm{~min}$ after the time of initial disturbance. We collected measures of adrenocortical responses to this standardized stress protocol twice for each bird, once when at $16 \mathrm{dph}$ and again at $198 \pm 5.9 \mathrm{dph}$. No blood samples were pooled for analyses.

To assess the adrenal responsiveness and negative feedback in the HPA axis, we administered ACTH challenges and Dex suppression tests on a separate day. The ACTH challenge measured the adrenals' capacity to secrete corticosterone in response to exogenous ACTH. The appropriate injection volume for each bird was calculated based on the $M_{\mathrm{b}}$ of the individual 1 day before the ACTH challenge. On the day of the ACTH challenge, baseline blood samples were collected, followed by an intramuscular injection of $25 \mathrm{IU} \mathrm{kg}^{-1}$ ACTH (Sigma-Aldrich, cat. no. A6303) delivered via a $300 \mu \mathrm{l}$ insulin syringe. After the injection, birds were held in an opaque bag and then blood sampled again $30 \mathrm{~min}$ after injection. Dex is a synthetic glucocorticoid that suppresses the endogenous secretion of glucocorticoids via negative feedback and has a low crossreactivity to the anti-corticosterone antibody used in radioimmunoassay. Consequently, Dex suppression tests measure the strength of negative feedback exerted on the HPA axis. After the capture and handling protocol, each bird was injected intramuscularly with $1000 \mu \mathrm{g} \mathrm{kg}^{-1}$ Dex (Sandoz Canada, Dexamethasone sodium phosphate injection USP, 2302; adjusted to $M_{\mathrm{b}}$ taken a day before). Following injection, birds were released into a cage and an additional blood sample was collected $30 \mathrm{~min}$ after injection. ACTH challenges and Dex suppression test were conducted when birds were $142 \pm 6.8$ and $198 \pm 5.9 \mathrm{dph}$, respectively.

Plasma corticosterone was quantified using a ${ }^{125}$ I radioimmunoassay (MP Biomedical) previously validated for zebra finch plasma (Schmidt and Soma, 2008). The standards ranged from 1.25 to $250 \mathrm{pg} \mathrm{tube}^{-1}$. The 


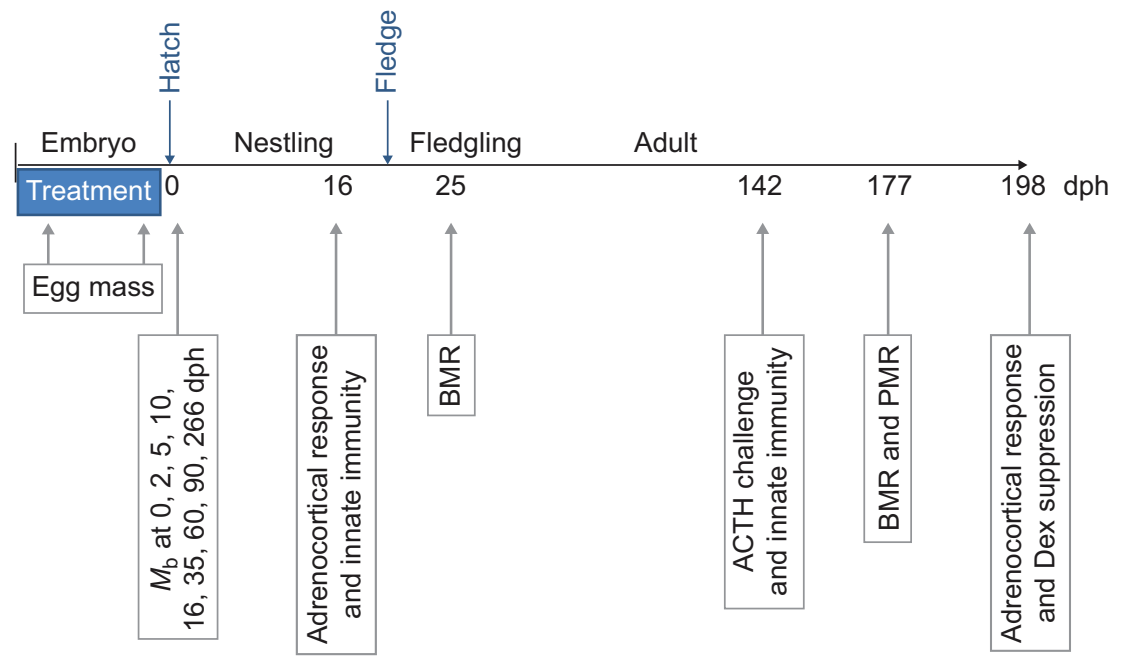

Fig. 6. Timeline of the experiment. Zebra finch eggs were incubated at $36.2,37.4$ or $38.4^{\circ} \mathrm{C}$ for the entire incubation period. Post-hatch environment was identical among the treatment groups.

interassay variations for high (125 $\left.\mathrm{pg} \mathrm{tube}^{-1}\right)$ and low $\left(12.5 \mathrm{pg}\right.$ tube $\left.\mathrm{e}^{-1}\right)$ controls were $6.36 \%$ and $9.32 \%$, respectively. The intra-assay variations for high and low controls were $4.78 \%$ and $11.53 \%$, respectively.

\section{Innate constitutive immunity}

To assess how incubation temperature affected innate constitutive immunity, we measured the antimicrobial capacity of whole blood against $C$. albicans (ATTC \#10231; Epower Microorganisms, cat. no. 0443E7, MicroBiologics) and E. coli (ATTC \#8739; Epower Microorganisms, cat. no. 0483E7, MicroBiologics) when the birds were, on average, 16 and $142 \pm 6.8 \mathrm{dph}$. All protocols were optimized for this species and age prior to analyses.

Antimicrobial activity against $C$. albicans is dependent on both plasma components and phagocytosis while killing of $E$. coli is dependent on plasma proteins only (Matson et al., 2006; Millet et al., 2007). We followed the method of Liebl and Martin (2009) with some modifications. Details of the assay are described in Kriengwatana et al. (2013). Briefly, sterile blood samples were collected within 5 min of entering the animal room. Antimicrobial assay for $C$. albicans was done immediately after blood collection while the assay for $E$. coli was done within 10 days of blood collection. The samples for the latter were kept on ice until frozen at $-80^{\circ} \mathrm{C}$. For the C. albicans killing assay, 1:48 dilution of whole blood to cell medium was used for $16 \mathrm{dph}$ samples while 1:96 dilution was used for adult $142 \mathrm{dph}$ samples. The primary incubation of whole blood, cell medium and C. albicans $\left[1 \times 10^{5}\right.$ colony forming units (CFU) $\mathrm{ml}^{-1}$ ] was $15 \mathrm{~min}$ at $30^{\circ} \mathrm{C}$. Sample duplicates, sample blank and positive and negative controls were incubated with tryptic soy broth for the second incubation at $30^{\circ} \mathrm{C}$. We used a nanodrop spectrophotometer (Nanodrop 2000c, Thermoscientific) to determine the absorbance at $300 \mathrm{~nm}$ after 24 to $48 \mathrm{~h}$ of secondary incubation. The time it took for the positive controls to reach an optimal absorbance varied between assays. Thus, we calculated the antimicrobial capacity as the average absorbance of sample duplicates/average absorbance of positive controls, each adjusted to its blank (\% microbial survival).

Antimicrobial activity against $E$. coli was assessed using the same protocol as for C. albicans, except that we used $1 \times 10^{4} \mathrm{CFU} \mathrm{ml^{-1 }}$ working solution, 1:6 dilution of blood to cell medium for both 16 and $\sim 142 \mathrm{dph}$, and $30 \mathrm{~min}$ primary incubation and $12 \mathrm{~h}$ secondary incubation at $37^{\circ} \mathrm{C}$.

\section{Metabolic rates}

We measured BMR in a subset of birds twice, when they were $25 \pm 1.7$ and $177 \pm 5.0 \mathrm{dph}$. BMR was measured using a flow-through respirometry system similar to that described in Gerson and Guglielmo (2011). The analyzer was calibrated daily with standard air containing $20.9 \% \mathrm{O}_{2}$ and $1.02 \% \mathrm{CO}_{2}$. Starting at 20:00 h, birds were weighed in a paper bag and placed in an airtight stainless-steel chamber inside an incubator. The incubator maintained the ambient temperature at $35^{\circ} \mathrm{C}$, which is within a thermoneutral zone for this species (Calder, 1964; Bech et al., 2004).
Birds fasted for $3 \mathrm{~h}$. Then the post-absorptive oxygen consumption from the remaining $9 \mathrm{~h}$ during the night cycle was used to analyze BMR. Incoming air was scrubbed of $\mathrm{CO}_{2}$ and water vapor and entered each chamber at constant rate of $\sim 350 \mathrm{ml} \mathrm{min}^{-1}$. Excurrent air was scrubbed of water vapor and subsampled for $\mathrm{CO}_{2}$ at a rate of $150 \mathrm{ml} \mathrm{min}^{-1}$, then subsampled for $\mathrm{O}_{2}$ after scrubbing of $\mathrm{CO}_{2}$ (Sable Systems, Las Vegas, $\mathrm{NV}$ ). Each chamber containing birds and a chamber sampling room air as a baseline were sampled for $10 \mathrm{~min}$ by a multiplexer every $70 \mathrm{~min}$. After the overnight measuring period, birds were weighed again then returned to the home cage. $\dot{V}_{\mathrm{O}_{2}}\left(\mathrm{ml} \mathrm{min}{ }^{-1}\right)$ was calculated from a $10 \mathrm{~min}$ interval with the lowest mean oxygen consumption among the measuring period after correcting for lab time using Expedata software (Sable Systems). The equation we used to calculate $\dot{V}_{\mathrm{O}_{2}}$ uses both fractional concentrations of $\mathrm{O}_{2}$ and $\mathrm{CO}_{2}$ [equations 10.6 and 10.7 in Lighton (2008)]. Here, we analyzed mass-corrected $\dot{V}_{\mathrm{O}_{2}}$, where $\dot{V}_{\mathrm{O}_{2}}$ was divided by the $M_{\mathrm{b}}$ of the individuals after the respirometry measurement $\left[\dot{V}_{\mathrm{O}_{2}}\left(\mathrm{ml} \mathrm{min}^{-1}\right) \mathrm{g}^{-1}\right]$.

PMR was measured $\sim 26 \mathrm{~h}$ after the end of the BMR measurement at $\sim 177 \mathrm{dph}$ using the same respirometry system. Birds were fasted for approximately $2 \mathrm{~h}$, then placed in a closed flight wheel $(16 \mathrm{~cm}$ width $\times 24 \mathrm{~cm}$ diameter) lined with rubber. The wheel was supplied with constant air of 3.5 liters $\mathrm{min}^{-1}$. The exiting air was subsampled at $\sim 285 \mathrm{ml} \mathrm{min}^{-1}$. Birds were left undisturbed for $5 \mathrm{~min}$ in the wheel covered with a cloth to acclimate. Beginning at 09:45 $\mathrm{h}$ and no later than $13: 30 \mathrm{~h}$, the wheel was spun manually by an experimenter who performed all the tests to encourage birds to hop and hover until the PMR was reached (generally occurred within $7 \mathrm{~min}$ ). Four ping-pong balls were placed inside of the wheel to prevent birds from walking. After reaching PMR, the experimenter kept the wheel spinning at the maximum speed at which birds could maintain the exercise for $2 \mathrm{~min}$. This method has been previously shown to estimate PMR in small passerines (Pierce et al., 2005; Price and Guglielmo, 2009). The PMR of each individual was calculated as a maximum average $\dot{V}_{\mathrm{O}_{2}}$ over a $15 \mathrm{~s}$ period corrected for $M_{\mathrm{b}}$ $\left[\dot{V}_{\mathrm{O}_{2}}\left(\mathrm{ml} \mathrm{min}{ }^{-1}\right) \mathrm{g}^{-1}\right]$.

\section{Statistical analyses}

All statistical analyses were performed using IBM SPSS 21. Data were transformed whenever necessary to meet the assumptions of normal distribution and homoscedasticity. All covariates whose $P$-value was higher than 0.1 were removed prior to the final analysis. Post hoc analysis was performed using Šidák adjustment.

\section{Egg measures}

Egg measures were analyzed using one-way ANCOVA. Egg mass loss (inverse transformed), incubation duration and hatchling mass were analyzed in 3 separate one-way ANCOVAs with incubation temperature as a main factor. For egg mass loss, incubation duration was used as a covariate and for hatchling mass, initial egg mass was also used as a covariate. 


\section{Survival}

Kaplan-Meier estimate with a log-rank test was used to compare survival among treatment groups. Survival due to temperature treatment during the incubation stage and survival after hatching (post treatment) was analyzed separately.

\section{Body mass}

$M_{\mathrm{b}}$ and physiological measures were analyzed using repeated measures ANCOVA and MANCOVA. Each sex was analyzed separately, excluding individuals that died before the juvenile stage. Growth, measured as increase in $M_{\mathrm{b}}$ (square-root transformed) over time, was analyzed using repeatedmeasures ANCOVA with an average of genetic parents' $M_{\mathrm{b}}$ (square-root transformed) just prior to pairing, as a covariate.

\section{Functioning of the hypothalamic-pituitary-adrenal axis}

We analyzed 6 parameters that reflected individuals' HPA axis function: baseline corticosterone levels at 16 and $198 \mathrm{dph}$, integrated stress response at 16 and $198 \mathrm{dph}$ and integrated responses to ACTH and Dex administration. Integrated responses were calculated as an area under the response curve and represent total corticosterone released during the monitored period. Baseline and integrated stress response collected at $16 \mathrm{dph}$ (log and square-root transformed, respectively) were analyzed in MANCOVA with $16 \mathrm{dph} M_{\mathrm{b}}$ as a covariate. Similarly, baseline and integrated stress response collected at $198 \mathrm{dph}$, integrated ACTH and Dex response at 140 and $198 \mathrm{dph}$ (log and square-root transformed) were analyzed in MANCOVA with $266 \mathrm{dph} M_{\mathrm{b}}$ as a covariate.

\section{Innate constitutive immunity}

Pearson correlation of microbicidal capacity against $C$. albicans and E. coli at 16 and $142 \mathrm{dph}$ revealed that only microbicidal capacity against C. albicans and E. coli at $142 \mathrm{dph}$ were marginally correlated $(P=0.093)$. Thus, data were analyzed together in MANOVA without data reduction. Arcsine square-root transformation was used for all the microbicidal capacity data.

\section{Metabolic rates}

Pearson correlation of BMR and PMR revealed that only BMR at 25 and $177 \mathrm{dph}$ were marginally correlated $(P=0.06)$. Thus BMR on 25 and $177 \mathrm{dph}$ as well as PMR at $177 \mathrm{dph}$ were analyzed together in MANOVA with incubation temperature as a fixed factor, without data reduction. Mean \pm s.e.m. is presented in the Results section and figures.

\section{Acknowledgements}

We thank Chris Guglielmo and Alexander Gerson for their help on the respirometry; Tara Farrell, Ainsley Furlonger, Zach Hall and Thom Luloff for help with blood sampling; Shawn Kubli, Dan Ardia, Sarah DuRant and Elizabeth MacDougall-Shackleton for their advice on and the use of space and the equipment for immune measures; Wayne Bezner-Kerr for his help on incubators; Carol Vleck for her advice on incubation temperatures in zebra finches; Tara Farrell for her help on statistical analysis; and Sean Aitken, Adrienne Berchtold and Jonathan Robertson for their help with bird care.

\section{Competing interests}

The authors declare no competing or financial interests.

\section{Author contributions}

H.W. and S.A.M. designed the study; H.W., B.K., N.A. and S.A.M. carried out the experiment; K.L.S. and K.K.S. analyzed hormone levels. H.W. and S.A.M. performed the statistical analyses. All authors contributed to manuscript preparation and approved the final version of the manuscript for publication.

\section{Funding}

Funding was provided by Natural Sciences and Engineering Research Council of Canada Discovery Grants to K.K.S. and S.A.M.-S.

\section{References}

Bech, C., Rønning, B. and Moe, B. (2004). Individual variation in the basal metabolism of Zebra finches Taeniopygia guttata: no effect of food quality during early development. Int. Cong. Ser. 1275, 306-312.

Bosque, C. and Bosque, M. T. (1995). Nest predation as a selective factor in the evolution of developmental rates in altricial birds. Am. Nat. 145, 234-260.
Burger, J., Zappalorti, R. T. and Gochfeld, M. (1987). Developmental effects of incubation temperature on hatchling pine snakes Pituophis melanoleucus. Comp. Biochem. Physiol. A Physiol. 87, 727-732.

Calder, W. A. (1964). Gaseous metabolism and water relations of the zebra finch, Taeniopygia castanotis. Physiol. Zool. 37, 400-413.

Case, T. J. (1978). On the evolution and adaptive significance of postnatal growth rates in the terrestrial vertebrates. Q. Rev. Biol. 53, 243-282.

Conway, C. J. and Martin, T. E. (2000). Effects of ambient temperature on avian incubation behavior. Behav. Ecol. 11, 178-188.

DuRant, S. E., Hepp, G. R., Moore, I. T., Hopkins, B. C. and Hopkins, W. A. (2010) Slight differences in incubation temperature affect early growth and stress endocrinology of wood duck (Aix sponsa) ducklings. J. Exp. Biol. 213, 45-51.

DuRant, S. E., Hopkins, W. A. and Hepp, G. R. (2011). Embryonic developmental patterns and energy expenditure are affected by incubation temperature in wood ducks (Aix sponsa). Physiol. Biochem. Zool. 84, 451-457.

DuRant, S. E., Hopkins, W. A., Hepp, G. R. and Walters, J. R. (2013). Ecological, evolutionary, and conservation implications of incubation temperature-dependent phenotypes in birds. Biol. Rev. Camb. Philos. Soc. 88, 499-509.

Eiby, Y. A. and Booth, D. T. (2009). The effects of incubation temperature on the morphology and composition of Australian Brush-turkey (Alectura lathami) chicks. J. Comp. Physiol. B 179, 875-882.

Fodor, A. and Zelena, D. (2014). The effect of maternal stress activation on the offspring during lactation in light of vasopressin. ScientificWorldJournal 2014 265394

Fowden, A. L., Giussani, D. A. and Forhead, A. J. (2006). Intrauterine programming of physiological systems: causes and consequences. Physiology 21, 29-37.

Gerson, A. R. and Guglielmo, C. G. (2011). House sparrows (Passer domesticus) increase protein catabolism in response to water restriction. Am. J. Physiol. Regul. Integr. Comp. Physiol. 300, R925-R930.

Guglielmo, C. G., McGuire, L. P., Gerson, A. R. and Seewagen, C. L. (2011). Simple, rapid, and non-invasive measurement of fat, lean, and total water masses of live birds using quantitative magnetic resonance. J. Ornithol. 152, 75-85.

Hepp, G. R., Kennamer, R. A. and Johnson, M. H. (2006). Maternal effects in Wood Ducks: incubation temperature influences incubation period and neonate phenotype. Funct. Ecol. 20, 308-314.

Krausova, T. and Peterka, M. (2007). Teratogenic and lethal effects of 2-24h hyperthermia episodes on chick embryos. J. Therm. Biol. 32, 193-203.

Kriengwatana, B., Wada, H., Macmillan, A. and MacDougall-Shackleton, S. A. (2013). Juvenile nutritional stress affects growth rate, adult organ mass, and innate immune function in zebra finches (Taeniopygia guttata). Physiol. Biochem. Zool. 86 769-781.

Liebl, A. L. and Martin, L. B.II. (2009). Simple quantification of blood and plasma antimicrobial capacity using spectrophotometry. Funct. Ecol. 23, 1091-1096.

Lighton, J. R. B. (2008). Measuring Metabolic Rates: A Manual for Scientists. New York: Oxford University Press.

Lundy, H. (1969). A review of the effects of temperature, humidity, turning and gaseous environment in the incubator on the hatchability of the hen's egg. In The Fertility and Hatchability of the Hen's Egg (ed. T. C. Carter and B. M. Freeman), pp. 143-176. Edinburth: Oliver and Boyd.

Martin, T. E. and Briskie, J. V. (2009). Predation on dependent offspring: a review of the consequences for mean expression and phenotypic plasticity in avian life history traits. Ann. N. Y. Acad. Sci. 1168, 201-217.

Matson, K. D., Tieleman, B. I. and Klasing, K. C. (2006). Capture stress and the bactericidal competence of blood and plasma in five species of tropical birds. Physiol. Biochem. Zool. 79, 556-564

Meaney, M. J. (2001). Maternal care, gene expression, and the transmission of individual differences in stress reactivity across generations. Annu. Rev. Neurosci. 24, 1161-1192.

Millet, S., Bennett, J., Lee, K. A., Hau, M. and Klasing, K. C. (2007). Quantifying and comparing constitutive immunity across avian species. Dev. Comp. Immunol. 31 188-201.

Nord, A. and Nilsson, J.-Å. (2011). Incubation temperature affects growth and energy metabolism in blue tit nestlings. Am. Nat. 178, 639-651.

Nord, A., Sandell, M. I. and Nilsson, J.-Å. (2010). Female zebra finches compromise clutch temperature in energetically demanding incubation conditions. Funct. Ecol. 24, 1031-1036

Olson, C. R., Vleck, C. M. and Vleck, D. (2006). Periodic cooling of bird eggs reduces embryonic growth efficiency. Physiol. Biochem. Zool. 79, 927-936.

Olson, C. R., Vleck, C. M. and Adams, D. C. (2008). Decoupling morphological development from growth in periodically cooled zebra finch embryos. J. Morphol. 269, 875-883.

Peterka, M., Peterková, R. and Likovský, Z. (1996). Teratogenic and lethal effects of long-term hyperthermia and hypothermia in the chick embryo. Reprod. Toxicol. 10, 327-332.

Pierce, B. J., McWilliams, S. R., O’Connor, T. P., Place, A. R. and Guglielmo, C. G. (2005). Effect of dietary fatty acid composition on depot fat and exercise performance in a migrating songbird, the red-eyed vireo. J. Exp. Biol. 208, 1277-1285.

Price, E. R. and Guglielmo, C. G. (2009). The effect of muscle phospholipid fatty acid composition on exercise performance: a direct test in the migratory white- 
throated sparrow (Zonotrichia albicollis). Am. J. Physiol. Regul. Integr. Comp. Physiol. 297, R775-R782.

Remeš, V. and Martin, T. E. (2002). Environmental influences on the evolution of growth and developmental rates in passerines. Evolution 56, 2505-2518.

Sakatani, M., Kobayashi, S.-I. and Takahashi, M. (2004). Effects of heat shock on in vitro development and intracellular oxidative state of bovine preimplantation embryos. Mol. Reprod. Dev. 67, 77-82.

Schmidt, K. L. and Soma, K. K. (2008). Cortisol and corticosterone in the songbird immune and nervous systems: local vs. systemic levels during development. Am. J. Physiol. Regul. Integr. Comp. Physiol. 295, R103-R110.
Sulik, K. K., Cook, C. S. and Webster, W. S. (1988). Teratogens and craniofacial malformations: relationships to cell death. Development 103, 213-231.

Vleck, C. M. (1981). Energetic cost of incubation in the Zebra Finch. Condor 83, 229-237.

Wingfield, J. C. (1994). Modulation of the adrenocortical response to stress in birds. In Perspectives in Comparative Endocrinology (ed. K. G. Davey, R. E. Peter and S. S. Tobe), pp. 520-528. Ottawa: National Research Council of Canada.

Zann, R. and Rossetto, M. (1991). Zebra finch incubation: brood patch, egg temperature and thermal properties of the nest. Emu 91, 107-120. 